\title{
Neuroendocrine control of body fluid homeostasis
}

S.M. McCann ${ }^{1}$,

J. Gutkowska ${ }^{2}$ and J. Antunes-Rodrigues ${ }^{3}$
1Pennington Biomedical Research Center (LSU), Baton Rouge, LA, USA ${ }^{2}$ Laboratory of Cardiovascular Biochemistry, Research Centre, CHUM, Masson Pavilion, Montreal, Quebec, Canada ${ }^{3}$ Departamento de Fisiologia, Faculdade de Medicina de Ribeirão Preto, Universidade de São Paulo, Ribeirão Preto, SP, Brasil

\section{Correspondence \\ S.M. McCann \\ Pennington Biomedical \\ Research Center (LSU) \\ 6400 Perkins Road \\ Baton Rouge, LA 70808-4124 \\ USA \\ Fax: +1-225-763-3030 \\ E-mail: mccannsm@mhs.pbrc.edu}

Research supported by NIH Grant MH51853 to S.M. McCann. Publication supported by FAPESP. ....................

Received May 24, 2002 Accepted November 8, 2002 ....................

\begin{abstract}
Angiotensin II and atrial natriuretic peptide (ANP) play important and opposite roles in the control of water and salt intake, with angiotensin II promoting the intake of both and ANP inhibiting the intake of both. Following blood volume expansion, baroreceptor input to the brainstem induces the release of ANP within the hypothalamus that releases oxytocin (OT) that acts on its receptors in the heart to cause the release of ANP. ANP activates guanylyl cyclase that converts guanosine triphosphate into cyclic guanosine monophosphate (cGMP). cGMP activates protein kinase $\mathrm{G}$ that reduces heart rate and force of contraction, decreasing cardiac output. ANP acts similarly to induce vasodilation. The intrinsic OT system in the heart and vascular system augments the effects of circulating OT to cause a rapid reduction in effective circulating blood volume. Furthermore, natriuresis is rapidly induced by the action of ANP on its tubular guanylyl cyclase receptors, resulting in the production of cGMP that closes $\mathrm{Na}^{+}$channels. The OT released by volume expansion also acts on its tubular receptors to activate nitric oxide synthase. The nitric oxide released activates guanylyl cyclase leading to the production of cGMP that also closes $\mathrm{Na}^{+}$channels, thereby augmenting the natriuretic effect of ANP. The natriuresis induced by cGMP finally causes blood volume to return to normal. At the same time, the ANP released acts centrally to decrease water and salt intake.
\end{abstract}

\section{Introduction}

Clinical studies involving patients with diabetes insipidus and the description of cases with brain lesions, which had either hypo- or hypernatremia suggested an important role for the hypothalamus in the control of water and salt intake and excretion. In the early fifties, Andersson and McCann (1) showed that microinjection of relatively large quantities of hypertonic saline into the hypothalamus of goats could induce drinking. The
Key words

- Oxytocin

- Vasopressin

- Atrial natriuretic peptide

- Nitric oxide

- Angiotensin II

- Guanylyl cyclase effect could also be obtained by microinjection of minute quantities of hypertonic saline into the hypothalamus, so as to produce drinking of water equal to a large percentage of the animal's body weight.

Since the effect was only repeatable two to three times, perhaps because of damage to the tissue by the hypertonic solution, electrical stimulation of the hypothalamus was performed (2). Electrical stimulation in the same region which evoked drinking following injection of hypertonic saline caused repro- 
ducible drinking with a very slight delay and with little after effects, such that the animals could drink a total of 30 to $40 \%$ of their body weight in water, sufficient to produce hypotonic hemolysis of the red cells and hemoglobinuria. The points of stimulation were in the medial and anterior hypothalamus extending from around the paraventricular nucleus down near the anterior ventral third ventricular $(\mathrm{AV} 3 \mathrm{~V})$ region. Some of these points of stimulation also evoked milk ejection via release of oxytocin (OT) and antidiuresis via release of antidiuretic hormone (vasopressin, VP); however, others produced only drinking and still others produced only the release of neurohypophyseal hormones.

Stimulation in or around the paraventricular nucleus, a site of origin of OT and vasopressinergic (VPergic) neurons, produced natriuresis (2). This was the first account of natriuresis from hypothalamic stimulation; however, it went largely unnoticed since the focus of the paper was on drinking, antidiuresis and milk ejection. This paper also provided the first evidence that both VP and OT are produced by neurons localized to the paraventricular as well as supraoptic nucleus. However, the preponderance of OT neurons is in the paraventricular nucleus and the preponderance of VPergic neurons is in the supraoptic nucleus (3). It is interesting to note that the area that induced natriuresis also is the site of cell bodies of the atrial natriuretic peptide (ANP) neurons (4).

We were unable to obtain survival of goats with lesions in this so-called "drinking area". Consequently, we made lesions in the medial hypothalamus surrounding the AV $3 \mathrm{~V}$ in dogs. These lesions produced complete adipsia but the animals tended to recover. During the adipsic period the animals would not drink water, but would drink fluid food in the form of milk or broth. During the adipsic period the animals were given water by gavage but still developed pronounced hypernatremia (5). The results were similar to those of Witt et al. (6), which were re- ported only in abstract form. Also, their lesions were complete ventral hypothalamectomies rather than the discrete small lesions induced by us. Prior to this time there was already evidence for a central nervous system $(\mathrm{CNS})$ role in natriuresis since a number of patients with brain lesions and hypernatremia had been reported (7). McCann et al. (8) observed hypernatremia in a number of cats with large lesions of the median eminence (ME) and ventral hypothalamus associated with diabetes insipidus. Consequently, they attributed the hypernatremia to dehydration. It is now known that VPergic diabetes insipidus in general is not associated with hypernatremia, so the hypernatremia observed was probably due in retrospect to decreased thirst, plus decreased secretion of ANP.

\section{Transmitters in the hypothalamus: control of fluid and electrolyte homeostasis}

Much later, Andersson et al. (9) microinjected hypertonic saline into the third ventricle $(3 \mathrm{~V})$ of goats and found that not only did it evoke reproducible drinking in the animals, in contrast to the difficulty of repeatability following injection into the tissue, but also evoked a marked natriuresis. This effect was confirmed following a $3 \mathrm{~V}$ injection of hypertonic saline into rats (10), and the role of various brain transmitters in the control of water, sodium chloride and food intake and of sodium excretion was then studied. Intraventricular injection of carbachol induced a dramatic, rapid 15-fold increase in water intake, whereas none of the other adrenergic or cholinergic drugs were effective (11), in agreement with earlier findings by Grossman (12) when injecting drugs into hypothalamic tissue. Both carbachol and isoproterenol, a ß-adrenergic agonist, evoked large increases in salt intake. Again, other drugs failed to produce significant effects.

Hypertonic saline injected into the $3 \mathrm{~V}$ 
produced a delayed increase in both water intake and food intake but did not alter salt intake. Therefore, it is clear that there is a cholinergic synapse in the pathways which mediate water intake, whereas both cholinergic and adrenergic synapses are involved in the mediation of salt intake $(11,12)$. Little further research along these lines has been undertaken.

The role of various transmitters in the natriuretic response to the $3 \mathrm{~V}$ injection of hypertonic saline was studied. Carbachol injection into the $3 \mathrm{~V}$ evoked a dramatic natriuretic response which mimicked the response to intraventricular hypertonic saline (13). Both natriuretic and kaliuretic responses and an increase in the sodium/potassium ratio were induced by intraventricular injection of norepinephrine or carbachol, whereas dopamine had no effect. The $\beta$-adrenoceptor stimulator, isoproterenol, induced an antinatriuretic and antikaliuretic effect. To determine the nature of the receptors involved, we injected adrenergic blockers and found that the $\alpha$-blocker, phentolamine, abolished the natriuretic response to intraventricular hypertonic saline and to norepinephrine and carbachol. In contrast, the $\beta$-adrenergic blocker, propranolol, induced natriuresis and kaliuresis when injected alone and had an additive effect when its injection was followed by that of norepinephrine or hypertonic saline. Propranolol also potentiated the natriuretic response to carbachol. Cholinergic blockade with atropine diminished the response to norepinephrine and blocked the natriuretic response to hypertonic saline. We suggested that sodium receptors in the ventricular wall (14) modify renal sodium excretion by a stimulatory pathway involving cholinergic and $\alpha$-adrenergic receptors and inhibit sodium excretion by a tonically active $\beta$-receptor pathway (15). Much later, $\beta$ receptors were described in the hypothalamus (16) and it was shown that their levels are modified by gonadal steroids (16).

Antunes-Rodrigues and his associates
(17) mapped the various pathways in the CNS controlling salt excretion and obtained similar results concerning the role of cholinergic and adrenergic receptors. Cholinergic or adrenergic stimulation of the medial septal area, medial preoptic area, anterior lateral hypothalamus, and subfornical organ as well as the anterior portion of the AV3V induced dose-related natriuresis accompanied by a lesser kaliuresis. Thus, considerable evidence indicates that the medial preoptic area, anterior lateral hypothalamus, subfornical organ, AV3V, habenula, stria medullaris, supraoptic nucleus, and medial septal area are organized in a neural circuit involved in the regulation of water and sodium intake and excretion. Specific hypothalamic lesions in this circuit altered salt intake (18). The role played by the CNS in the control of renal sodium excretion has been demonstrated by other authors as well (14).

\section{Natriuretic hormones}

During the sixties much attention was paid to the possibility of the existence of a natriuretic hormone. The idea stemmed from the experiments of DeWardener and Clarkson (19) showing that natriuresis could occur following body fluid expansion even though factors such as increased glomerular filtration rate, or changes in aldosterone secretion were eliminated. Davis and Freeman (20) obtained evidence for a circulating natriuretic factor in volume-expanded dogs by crosscirculation experiments.

The Czech group (21) reported the purification of a hypothalamic natriuretic factor and claimed it was an OT analog. Consequently, we began to work with Orias $(22,23)$ on this problem. We confirmed that both VP and OT are natriuretic. Also, $\alpha$ - and $\beta$-melanocyte-stimulating hormone (MSH) had natriuretic activity in conscious water-loaded male rats. It is now known that $\alpha-\mathrm{MSH}$ is produced in neurons in the brain, and is also released from the intermediate lobe of the 
pituitary, so it was possible that the natriuretic hormone is MSH. However, it is still not clear if MSH has a physiologic role in the induction of natriuresis.

Since there was considerable evidence suggesting that there was a natriuretic hormone in the hypothalamus and that it could be related to OT, we evaluated the effect of $\mathrm{ME}$ lesions on the natriuretic responses to hypertonic saline and carbachol or norepinephrine injected into the $3 \mathrm{~V}$, since if indeed there were a hypothalamic natriuretic hormone, it might be expected to gain exit to the general circulation via the neurohypophysis. These animals had lesions that destroyed most of the ME and thereby induced diabetes insipidus because of interruption of the supraopticohypophyseal tract and consequent elimination of VP and OT secretion. These $\mathrm{ME}$ lesions blocked the natriuresis, kaliuresis and antidiuresis, which followed the injection of hypertonic saline or norepinephrine into the $3 \mathrm{~V}$. Sham lesions did not block the responses (24). Hypophysectomy did not block the responses, findings that ruled out the participation of anterior pituitary hormones (24). With Orias, we had shown that the responses still occurred in rats with hereditary diabetes insipidus that lacked VP (25). Thus, although natriuretic, both VP and MSH were eliminated as essential components of the natriuretic responses. Therefore, we suggested that these lesions had interrupted the secretion of a natriuretic hormone involved in the induction of central natriuresis.

\section{Atrial natriuretic peptide}

We were amazed to become aware of the discovery of ANP (26) in 1981. It had been known even when McCann was with Andersson in Sweden that dilation of the atria could produce diuresis from the pioneering experiments of Gauer and Henry (27). At that time it was thought that distension of the atria activated impulses which traveled up the vagus to inhibit the release of antidiuretic hormone. The decreased release of this hormone was thought to be responsible for the diuresis. Water immersion to the neck in baths had been known to evoke diuresis since the mid 19th century. Immersion probably increased venous return to the heart and dilated the atria.

Therefore, it was a great shock to find that this was not due to a reflex activation of the brain but rather to the release of ANP from the right atrium of the heart. Space does not permit a description of the history of ANP; however, the thinking then changed to the idea that natriuresis following volume expansion was due to secretion of this peptide from the atria which circulated to the kidneys and evoked natriuresis (28).

\section{The brain ANPergic neurons}

The demonstration of ANP in extracts from various hypothalamic regions (29) and the evidence that ANP had opposite actions to those of angiotensin II (AII) at every site so far studied (30) led McCann to hypothesize that ANP might have opposite actions to those of AII in control of water intake and that it might be the long sought hypothalamic natriuretic hormone. Indeed, it is now known that ANPergic neurons are localized in the region extending from the paraventricular nucleus rostrally to the organum subfornicalis and ventrally to the organum vasculosum lamina terminalis, areas known to be implicated in thirst (31), and that their axons also project down to the ME and neural lobe (4). There they terminate in proximity to either the long or short portal vessels, so that the peptide could be transported to the anterior pituitary and also into the general circulation.

\section{The ANPergic neurons in water and salt intake}

Injection of ANP into the $3 \mathrm{~V}$ of waterdeprived rats induced a dose-related inhibi- 
tion of drinking with doses of 1.0 or $2.0 \mathrm{nmol}$ ANP. Inhibition could only be obtained following intravenous injection of the peptide at the higher $2.0 \mathrm{nmol}$ dose, indicating a central action of the intraventricularly injected peptide (32). ANP could also block AII-induced drinking. This inhibitory response was present at doses of intraventricularly injected AII ranging from 4.8 to 25 pmol; however, the $1.0 \mathrm{nmol}$ dose of ANP given 5 min before AII infusion was unable to block responses to much higher doses of AII ranging from 96 to $956 \mathrm{pmol}$ (32).

Since AII also increases salt intake, we speculated that ANP would have the opposite effect and inhibit saline intake when injected into the $3 \mathrm{~V}$ of conscious, salt-depleted rats. Animals were salt-depleted by 4 days of salt restriction followed by peritoneal dialysis with 5\% glucose solution. Salt intake was suppressed dramatically by a minimal effective dose of $0.2 \mathrm{nmol}$ ANP. There was no additional effect with a 10 -fold higher dose. The suppression was maintained during the $24 \mathrm{~h}$ after central injection of the peptide; however, in this case the inhibition was somewhat greater with the higher dose of ANP. In contrast, the relatively low intake of distilled water that was also offered to the animals was not affected by any dose of ANP (33). Consequently, it appears that this peptide can drastically suppress dehydration and AII-induced drinking and is even more potent in suppressing salt intake.

To evaluate the physiologic significance of various peptides in the control of water intake, we injected into the $3 \mathrm{~V}$ highly purified antibodies against peptides thought to be involved and injected control animals with normal rabbit serum in the same volume. Since the evidence was already quite strong that AII may be involved in the drinking which follows hemorrhage and depletion of extracellular fluid volume, we decided to evaluate its possible role in dehydrationinduced drinking. Antiserum directed against AII was microinjected into the $3 \mathrm{~V}$ of rats that had been deprived of water overnight. We had previously found that frequently there is a delay following intraventricular injection of antiserum against peptides before they are effective. These delays may represent time for the antiserum to be absorbed from the ventricle and to diffuse to the site of action of the peptide. In this case, if water was offered immediately after injection of the antiserum, drinking was not altered. If water was offered $1 \mathrm{~h}$ after injection, drinking was largely blocked. At $3 \mathrm{~h}$ after injection of the antiserum, drinking was completely abolished. These results indicate that AII is required to induce the drinking which follows dehydration (34).

Previous attempts had been made to block dehydration-induced drinking with saralasin, an antagonist of AII; however, except for one experiment in the rat in which lateral ventricular infusion of saralasin commencing 30 min prior to giving the animals access to water partially suppressed drinking (35), these experiments with saralasin have been negative (36). These results contrast strikingly with the dramatic effectiveness of AII antiserum in blocking dehydration-induced drinking. We believe that the discrepancy is probably related to the short duration of action of saralasin, plus the failure to distribute it to the active sites following infusion into the lateral ventricle which would not distribute the antagonist uniformly bilaterally and therefore might not completely inactivate the AII receptors. We conclude that AII, either reaching the brain via the circulation and uptake via the circumventricular organs, or more likely released from neurons containing AII within the hypothalamus, which have been found in close association with ANP neurons, plays an essential role in dehydration-induced drinking. By contrast, AII appears to play no role in the normal prandial drinking that occurs concomitantly with feeding when the lights are turned off, because the antiserum injected $3 \mathrm{~h}$ before lights off had no effect on prandial drinking (34). 


\section{Brain ANPergic neuronal system and release of ANP}

The next question was the role of the brain ANPergic neuronal system in CNSinduced natriuresis brought on by intraventricular injection of hypertonic saline or activation of brain cholinergic and adrenergic circuits by carbachol or norepinephrine. We had earlier thought that these effects were brought about by the release of a hypothalamic natriuretic hormone. We hypothesized that they were brought about by neural activation of the release of ANP from the neurohypophysis or the atria.

Therefore, we evaluated the possible role of brain ANP in evoking the changes in renal sodium excretion that followed stimulations or lesions of the $A V 3 \mathrm{~V}$, a region further implicated in control of sodium excretion by later experiments (37). Injection of carbachol into the AV3V produced the expected natriuresis on the basis of our earlier experiments, which was accompanied by a dramatic rise in plasma ANP concentration and a rise in ANP content in the medial basal hypothalamus, the neurohypophysis and particularly the anterior hypophysis but without alterations in the content of ANP in the lungs or the right or left atrium (38). The marked elevations in content of the peptide in the basal hypothalamus and neuro- and adenohypophysis suggested that the natriuresis resulting from this stimulation is brought about at least in part by release of ANP from the brain.

Conversely, there was a dramatic decline in plasma ANP at both 24 and $120 \mathrm{~h}$ after AV3V lesions had been placed (39). This was accompanied by a slight decline in the content in the right atrium at $24 \mathrm{~h}$ after lesions, but there was a significant increase at $120 \mathrm{~h}$. These small changes contrasted sharply with the dramatic decline in the content of the peptide in the medial basal hypothalamus, ME, neurohypophysis, choroid plexus, anterior hypophysis, and olfactory bulb. These declines persisted or became greater at $120 \mathrm{~h}$, except in the olfactory bulb in which the decline was no longer significant.

Therefore, lesions which destroyed the perikarya of ANPergic neurons caused a decline in ANP content in presumed projection areas of these neurons to the olfactory bulb, where they are probably involved in control of salt and water intake (40). ANP content of the choroid plexus also declined, probably because of the loss of input from the AV3V neurons. There is evidence that the ANP neuronal projection to the choroid plexus may be involved in cerebrospinal fluid formation (40). Destruction of the AV3V also caused loss of ANP from caudal axonal projections to the neurohypophysis, whereas only a delayed increase in the left atrial ANP content occurred, probably related to decreased release of the peptide in the presence of continued synthesis which led to increased tissue content.

The dramatic decline in plasma ANP after AV3V lesions was accompanied by a very dramatic decline in content of ANP in regions containing the caudal axonal ANP neuronal connections to the ME and neural lobe of the pituitary gland, that was probably caused by the release of these stores of the peptide that could not be replenished by axoplasmic flow of the peptide from the destroyed perikarya.

In view of the much larger quantities of the peptide stored in the atria, it is probable that changes in atrial release contribute to the alterations in plasma ANP observed after stimulation or ablation of the $\mathrm{AV} 3 \mathrm{~V}$ region; however, these results suggested that the dramatic changes in plasma ANP that followed these manipulations may be due to altered release of the peptide from brain structures as well as the atria and lungs $(38,39)$. These stimulation and lesion experiments support a crucial role of the CNS in controlling ANP release. 


\section{Role of hypothalamic ANPergic neurons in volume expansion- induced release of ANP}

Blood volume expansion (BVE) causes a release of ANP that is believed to be important in induction of the subsequent natriuresis and diuresis which, in turn, acts to reduce the increase in blood volume. Since stimulation of the AV3V induced a rapid elevation of plasma ANP, whereas lesions of the AV3V were followed by a marked decline in plasma concentration of the peptide, we hypothesized that release of ANP from the brain ANP neuronal system might be important to the control of plasma ANP. As already described, the perikarya of the ANP-containing neurons are densely distributed in the $\mathrm{AV} 3 \mathrm{~V}$ and their axons project to the ME and neural lobe $(4,31)$.

To test the hypothesis that these neurons are involved in volume expansion-induced ANP release, we destroyed the AV3V, the site of the perikarya, in male rats by electrolytic lesions. Other lesions were made in the $\mathrm{ME}$ and posterior pituitary, sites of termination of the axons of these neurons, and also hypophysectomy was performed in other animals (39).

In conscious freely moving animals, volume expansion and stimulation of postulated sodium receptors (14) in the hypothalamus were induced by injection of hypertonic $\mathrm{NaCl}$ solution $(0.5$ or $0.3 \mathrm{M} \mathrm{NaCl}, 2 \mathrm{ml} / 100$ $\mathrm{g}$ body weight). Volume expansion alone was induced with the same volume of an isotonic solution $(\mathrm{NaCl}$ or glucose). In the sham-operated rats, volume expansion with hypertonic or isotonic solutions caused equivalent rapid increases in plasma ANP that peaked at $5 \mathrm{~min}$ and returned nearly to control values by $15 \mathrm{~min}$. Lesions caused a decrease in the initial levels of plasma ANP compared with values from the sham-operated rats, and each type of lesion induced a highly significant suppression of the response to volume expansion on testing 1-5 days after lesions were made.

Because a common denominator of the lesions was elimination of the brain ANP neuronal system, these results suggest that brain ANP plays an important role in the mediation of the release of ANP that occurs after volume expansion. Since the content of ANP in this system is 1000 -fold less than that in the atria, it is likely that release of brain ANP associated with this stimulus cannot account for the 4-fold increase in plasma ANP within $5 \mathrm{~min}$ of volume expansion. Therefore, a large increase in release from the atrium must occur. This could be mediated by efferent neural input to the heart, but this is unlikely since neurolobectomy blocked not only volume expansion-induced ANP release, but also the release of other neurohypophyseal hormones, such as VP, OT or endothelin. Thus, these neurohypophyseal hormones may induce release of ANP from atrial myocytes $(39,40)$.

In other experiments, we determined the essentiality of the brain ANP neuronal system to the BVE-induced ANP release induced by hypertonic saline by injection of antiserum directed against ANP into the AV3V prior to inducing volume expansion. The antiserum had no effect on resting levels of ANP; however, it partially blocked the increase in ANP and the natriuresis that followed BVE (41). Other experiments in sheep had given similar results (42). Therefore, it appears that the essentiality of the nervous system in these responses of ANP to volume expansion is conferred by the ANP neuronal system.

We had previously shown that cholinergic and adrenergic synapses within the hypothalamus mediated the natriuresis induced by $3 \mathrm{~V}$ injection of hypertonic saline (15). Therefore, we evaluated their role in the ANP release evoked by volume expansion. The receptor-blocking agents were injected into the $3 \mathrm{~V} 30 \mathrm{~min}$ prior to BVE as previously described. These blockers had no effect on resting levels of the hormone just 
prior to BVE; however, a highly significant blockade of the responses was induced by the prior injection of the muscarinic cholinergic receptor blockers, atropine sulfate ( 5 $\mathrm{nmol}$ in $2 \mu \mathrm{l} 0.9 \% \mathrm{NaCl}$ ) or methyl atropine at a similar dose. Microinjection of the $\alpha$ receptor blocker, phentolamine (5 $\mathrm{nmol}$ in 2 $\mu 1$ saline) also markedly suppressed the ANP response (43).

To determine whether this was a central or possibly a systemic effect of the blockers, methyl atropine $(0.01 \mathrm{nmol} / 100 \mathrm{~g}$ body weight), which does not cross the bloodbrain barrier, was injected intraperitoneally (ip) $30 \mathrm{~min}$ before volume expansion. Atropine also had no effect on basal levels of plasma ANP, but in striking contrast to the blockade of the response to volume expansion induced by intraventricular injection of methyl atropine, the response to volume expansion was markedly enhanced by ip injection of methyl atropine. The results therefore indicate that hypothalamic muscarinic and $\alpha$-adrenergic synapses are essential for ANP release in response to volume expansion (43). The ability of methyl atropine, a blocker of peripheral cholinergic receptors, to augment the response suggests an inhibitory role of vagal cholinergic efferents in ANP release.

Thus, the results obtained up to that point indicated the crucial participation of the CNS and the brain ANP neurons in the response of ANP and natriuresis to volume expansion. We considered the possibility that the baroreceptors, when stretched by volume expansion, would activate the brain ANP neurons, which would then produce the release of ANP and the ensuing natriuresis. Therefore, we determined the role of the baroreceptors in affecting the increase in plasma ANP from volume expansion induced by intravenous injection of hypertonic saline solution $(0.3 \mathrm{M} \mathrm{NaCl}, 2 \mathrm{ml} / 100 \mathrm{~g}$ body weight, over $1 \mathrm{~min}$ ) into conscious, freely moving male rats (44). In sham-operated rats, BVE induced a rapid increase in plasma
ANP as before. The concentration peaked at $5 \mathrm{~min}$ and remained elevated at $15 \mathrm{~min}$ after saline injection. One week after deafferentation of the carotid-aortic baroreceptors, basal plasma ANP concentrations were highly significantly decreased on comparison with values for sham-operated rats; plasma ANP levels were greatly reduced 5 min after BVE in the deafferented rats. Unilateral right vagotomy reduced resting levels of plasma ANP but not the response to BVE; resting concentrations of plasma ANP and responses to expansions were normal in bilaterally vagotomized rats. In rats that had undergone renal deafferentation, resting levels of ANP were normal but the response to BVE was significantly suppressed.

The evidence indicated that afferent impulses via the right vagus nerve may be important under basal conditions, but are not required for the ANP release induced by BVE. In contrast, baroreceptor impulses from the carotid-aortic sinus regions and the kidney are important pathways involved in neuroendocrine control of ANP release. Others have also found that the carotid-aortic baroreceptors are important in mediating the response (45). The evidence from these experiments and our previous stimulation and lesion studies indicates that the ANP release in response to volume expansion is mediated by afferent baroreceptor input to the AV3V region, which mediates the increased ANP release via activation of the hypothalamic ANP neuronal system (44).

\section{Role of the locus ceruleus and raphe nuclei in transmission of afferent input to the AV3V region}

Since baroreceptor afferents terminate in the nucleus tractus solitarius (NTS), we hypothesized that baroreceptor impulses to the NTS might be relayed to the locus ceruleus which would then transmit the information to the AV3V region by axons of the noradrenergic neurons located there. Indeed, lesions 
of the locus ceruleus lowered resting ANP levels and blocked the response of ANP to volume expansion (Franci J and AntunesRodrigues J, unpublished data). We speculate that the axons of these noradrenergic neurons projecting to the AV $3 \mathrm{~V}$ region activate cholinergic interneurons there, which in turn stimulate the hypothalamic ANPergic neurons. These neurons would activate efferent neurohumoral or neural pathways, which induce the release of ANP from the brain and in much greater quantities from the atria.

An afferent pathway to the AV $3 \mathrm{~V}$ region via serotonergic neurons with cell bodies in the raphe nuclei has been demonstrated (46). Therefore, we hypothesized that serotonin (5-HT) may play a role in the control of ANP neurons in the region of the AV $3 \mathrm{~V}$. Indeed, earlier studies had shown that injection of 5HT agonists into the third or lateral ventricle could increase plasma ANP, and that 5- $\mathrm{HT}_{2}$ receptor blockers $(47,48)$ prevented the responses. To determine the effect of loss of 5 HT input into the AV3V region, bilateral lesions were placed in the dorsal raphe nuclei (DRN), a major source of 5-HT neurons that project to the $\mathrm{AV} 3 \mathrm{~V}$ region, and in other animals, depletion of 5-HT from serotonergic neurons was obtained by systemic administration of parachlorophenylalanine (PCPA), an amino acid that competes with tryptophan, the substrate of tryptophan hydroxylase, the rate-limiting enzyme in the synthesis of indolamines (49).

Rather surprisingly at first glance, the DRN lesions produced a diabetes insipiduslike state in which there was a highly significant increase in water intake and urine volume beginning on the 1st day after the lesions, reaching a peak of water intake at 3 days, followed by a gradual decline in water intake and urine volume to control levels one week after the lesions had been placed. During diuresis, urine osmolality was dramatically reduced, as was sodium excretion. When the animals were water-loaded and sodium excretion was measured on day 2 after the lesions, sodium excretion was drastically reduced, although it did recover by 4 and 14 days after the lesions.

We believe that these changes were due to a drastic suppression of ANP release since the basal levels of ANP were highly significantly reduced and we concluded that the serotonergic system has a tonic stimulatory effect on the release of ANP. When this ANP drive is removed by the lesions, there may be a removal of tonic inhibition by ANP of AIIsecreting neurons within the AV3V region resulting in increased AII release which then brings about an increase in water intake. At the same time, the reduction in ANP output causes a reduction in renal sodium excretion. Consequently, water intake is increased, leading to a reduction of VP release and to hypotonic urine with drastically reduced sodium concentration. The animals recovered, probably because the DRN is not the only source of serotonergic input, which is also delivered through the median raphe nuclei. Depletion of 5-HT from these serotonergic cells also produced a similar picture (49).

When the rats with PCPA lesions were water-loaded 5 days after the lesions, they showed a reduction in natriuresis similar to that of the rats with DRN lesions. The results differed from those in rats with DRN lesions only in that there was also a significant reduction in kaliuresis in the PCPA-injected rats. These effects were probably also due to reduced stimulation of the ANP neurons with a resultant reduction in plasma ANP. As in the case of the DRN lesions, not only the initial levels of plasma ANP, but also the response of plasma ANP to BVE were significantly reduced, although the reduction was not complete, as was the case for the DRN lesion group.

Therefore, we concluded that there was a tonic stimulatory input from the 5-HT neurons to the hypothalamic ANP neurons which, when removed, resulted in disinhibition of AII release causing increased water intake 
and decreased ANP release into the circulation resulting in sodium retention. The raphe nuclei may be stimulated by afferent input from the baroreceptors via the NTS and this then may be responsible in part for the stimulation of ANP release, which occurs following volume expansion. Alternatively, a tonic stimulatory drive via these neurons may be all that is required to have the volume expansion-induced release of ANP, and the major stimulation may be via the locus

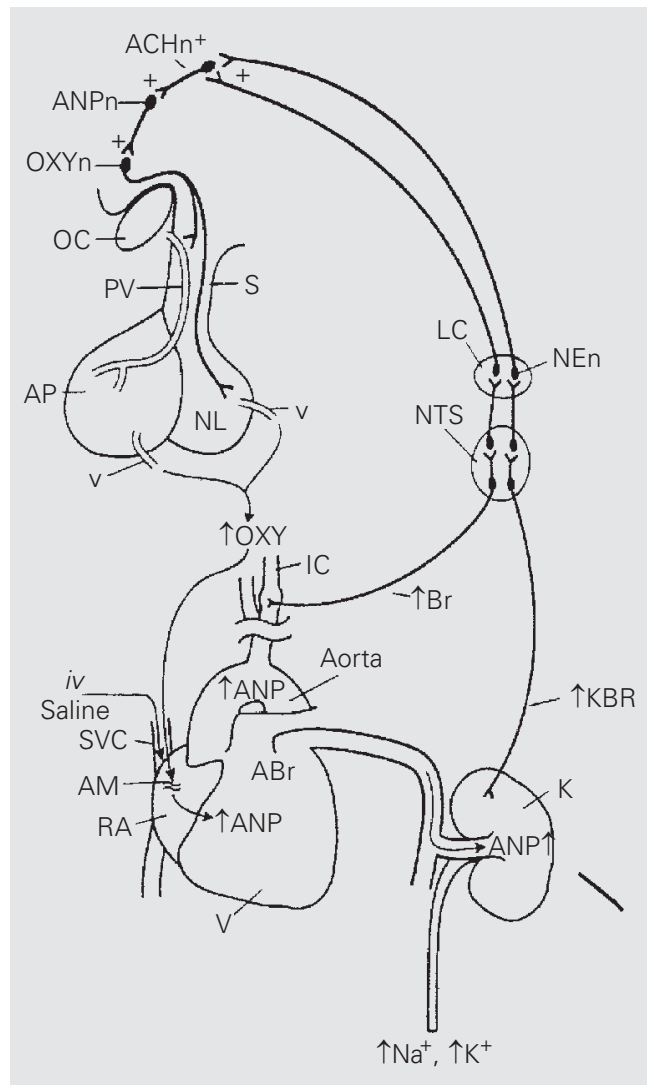

Figure 1. Schematic diagram of the mechanism of natriuresis following blood volume expansion by intravenous (iv) injection of isotonic saline into the right atrium. OXYn, oxytocinergic neuron; $\mathrm{ACHn}$, acetylcholinergic neuron; NEn, norepinephrine neuron; ANP, atrial natriuretic peptide; ANPn, ANPergic neuron; OC, optic chiasm; PV, portal vessel; AP, anterior lobe of the pituitary gland; NL, neural lobe of the pituitary gland; $v$, vein; LC, locus ceruleus; NTS, nucleus tractus solitarius; IC, internal carotid artery; RA, right atrium; $\mathrm{V}$, ventricles; $\mathrm{Br}$, baroreceptor afferents; KBR, renal baroreceptor afferents; K, kidney; S, pituitary stalk; AM, atrial myocyte; SVC, superior vena cava (from Ref. 51, with permission). ceruleus with increased noradrenergic drive to the AV3V region. Further work will be necessary to distinguish between these two possibilities.

We have illustrated (Figure 1) the putative pathway of activation of ANP release and natriuresis via volume expansion, which involves distension of baroreceptors in the right atria, carotid sinuses and aortic arch and in the kidney, altering their afferent input to the brainstem in the NTS. Impulses from the NTS activate the locus ceruleus since lesions of the locus ceruleus, a major source of noradrenergic axons to the hypothalamus, reduced resting ANP levels and blocked the response to volume expansion. The axons of these noradrenergic neurons stimulate hypothalamic cholinergic interneurons there, which in turn stimulate the hypothalamic ANPergic neurons. These neurons activate efferent neurohumoral or neural pathways that induce the release of ANP from the brain and the atria.

\section{Efferent pathways by which volume expansion stimulates ANP release}

Some of the ANP neurons terminate in the ME and neural lobe of the hypophysis. It is probable that their activation leads to release of the peptide into the vasculature draining the ME and the neural lobe. Since the quantity of ANP is more than a 1000 -fold less in these structures than in the atria (38), we believe that ANP released from the brain plays a minor role in the responses. Rather, we would suggest that these ANPergic neurons activate descending pathways that then activate efferent pathways to the heart with consequent release of ANP from cardiac myocytes. Combined release from both sources then accounts for the increase in plasma ANP concentrations that mediates the ensuing natriuresis. We do not believe that the efferent pathway to the heart is principally neural. It cannot be cholinergic since bilateral section of the vagi does not 
block the response to volume expansion. It is unlikely that it is a sympathetic efferent pathway since volume expansion by elevating blood pressure should, if anything, diminish sympathetic outflow. There is a possibility that there could be an unknown efferent pathway reaching the atria, perhaps peptidergic in nature, or even nitricoxidergic.

Instead, we believe that release of brain peptides induced by ANP neurons is probably the major pathway. These peptides circulate to the atria and act directly on atrial myocytes to stimulate the release of ANP. Because a large amount of endothelin was discovered in the neural lobe, we evaluated the possibility that this could be the activator of the release of ANP by atrial myocytes. However, our data indicate that this is unlikely (50). $\alpha-\mathrm{MSH}$ is also natriuretic and we evaluated its effect on ANP release, but so far the results have not been impressive. The major peptides of the neurohypophysis are VP and OT. Both are natriuretic in the rat, but OT is by far the most potent of these natriuretic peptides and in our earlier experiments we considered the possibility that OT was indeed the natriuretic peptide (24).

Therefore, we reevaluated the role of OT in the natriuresis and ANP release induced by volume expansion (51). OT (1-10 nmol) injected ip in water-loaded conscious rats caused significant dose-dependent increases in urinary osmolality, natriuresis and kaliuresis, results exactly similar to those obtained by volume expansion in the conscious water-loaded rat under the same conditions as used in all our prior experiments (41). Plasma ANP concentrations increased nearly 4-fold $20 \mathrm{~min}$ after the $10 \mathrm{nmol}$ dose of OT, but there was no change in plasma ANP values in control animals. OT (1 or $10 \mathrm{nmol})$ injected $i v$ induced a dose-related increase in plasma ANP peaking at $5 \mathrm{~min}$. Therefore, we have demonstrated that OT can indeed induce natriuresis and kaliuresis in the quantities seen with volume loading and furthermore that it induces a concomitant release of
ANP with the same time course as that we have previously found with BVE.

To determine if indeed BVE induces OT as well as ANP release, intra-atrial injections of isotonic saline were given $(2 \mathrm{ml} / 100 \mathrm{~g}$ body weight), which induced a rapid (5 min post-injection) increase in plasma OT and ANP concentrations and a concomitant decrease in plasma arginine VP concentrations (51). When hypertonic volume expansion was produced by injection of $0.3 \mathrm{M} \mathrm{NaCl}$, which should also stimulate putative osmoor sodium receptors and might be expected to cause a secretion of VP, there was a greater increase in plasma ANP and also OT, but not significantly different from the increases in the isotonic volume-expanded animals. However, in contrast to isotonic volume expansion, there was a transient (5 min) increase in plasma arginine VP.

Consequently, we have developed the hypothesis that baroreceptor activation of the CNS by BVE stimulates the release of OT from the neurohypophysis (Figure 1). This OT circulates to the right atrium to induce release of ANP. ANP circulates to the kidney and induces natriuresis and diuresis, which restores body fluid volume to normal levels. We showed that suckling, which causes the release of OT, increases plasma ANP in rats, and that an OT antagonist injected $15 \mathrm{~min}$ prior to suckling completely blocked the increase in ANP caused by suckling (51).

It has been noteworthy throughout all of these studies that smaller changes in potassium excretion parallel those in sodium. We found this also to be the case with injection of OT. Similarly, ANP produces a greater natriuresis but a significant kaliuresis (51). Consequently, we concluded that OT and ANP can account for the decrease in potassium excretion when there is a reduction in ANP release. This view may need modification with the recent discovery of a kaliuretic peptide, which produces kaliuresis with little or no natriuresis, and is the 20 -amino acid peptide 
amino terminal extension of ANP (52).

Furthermore, it has now been shown in man that water immersion to the neck, as would be expected from results obtained even in the middle ages, produces diuresis, natriuresis and kaliuresis. Vesely et al. (53) have shown that in this situation, there is an elevation of plasma concentrations of both kaliuretic peptide and ANP. However, the time course of the ensuing kaliuresis is more easily explained on the basis of increased concentrations of kaliuretic peptide than ANP. Therefore, it is possible that stimuli that either increase or decrease plasma ANP also produce a concomitant stimulation or inhibition of the release of kaliuretic peptide. Further experiments are needed to determine whether both peptides are involved in the changes or only ANP.

\section{Role of oxytocin, ANP and nitric oxide in the cardiovascular system and kidney}

Supporting a direct action of OT on the heart, incubation of quartered rat atria (54) or perfusion of rat hearts (55) with OT caused ANP release in a dose-related fashion. OTstimulated ANP release from both experimental preparations was blocked by an OT antagonist $(54,55)$. Furthermore, OT decreased the rate and force of contraction of the heart in both experimental preparations and these effects were not blocked by atropine at a concentration that blocks muscarinic cholinergic actions on the heart (54). These findings led to the hypothesis that OT evoked ANP release from the heart by action on putative OT receptors (OTR).

OTR have been characterized in the rat heart that appear to be identical to those in other organs (56). The presence of OTR in the rat heart was demonstrated by autoradiography performed on frozen sections. Increasing quantities of unlabeled OT progressively inhibited the radiolabeled OT antagonist binding. OTR binding affinity was simi- lar in atrial and ventricular receptors, with $K_{\mathrm{d}}$ of $\sim 71$ and $\sim 30 \mathrm{pM}$, respectively. The number of OTR was also similar in all heart compartments.

The presence of specific transcripts for OTR in the rat heart was demonstrated by polymerase chain reaction (PCR) amplification of cDNA obtained from mRNA of both rat atria and ventricles by using specific oligonucleotide primers. The amplified rat heart OTR sequence was identical to that of the rat uterus and hypothalamus, indicating a close structural relationship. The PCR amplification product obtained had the expected molecular size of $373 \mathrm{bp}$, the same receptor size as found in the rat uterus. Reverse transcription (RT)-PCR did not show any extra band that could indicate the presence of different splicing forms. The presence of OTR transcripts was also shown by in situ hybridization in atrial and ventricular tissues using reverse RT-PCR primers as probes (55).

The functionality of heart OTR was demonstrated by the ability of OT to release ANP from the isolated perfused heart (55). OT $(1 \mu \mathrm{M})$ addition to the perfusion buffer significantly enhanced ANP release. OT $(1 \mu \mathrm{M})$ resulted in a gradual and significant decrease in heart rate from $330 \pm 12$ to $288 \pm 9 \mathrm{bpm}$. The OT antagonist inhibited ANP release in a dose-related manner. Interestingly, the OT antagonist at the concentration of $1 \mu \mathrm{M}$ reduced ANP release below control levels. This observation implied the existence of intracardiac synthesis and release of OT.

Indeed, OT is present and synthesized in the heart $(56,57)$. OT has been detected by radioimmunoassay in all four chambers of the rat heart. The highest concentration was found in the right atrium $(2,128 \pm 114 \mathrm{pg} / \mathrm{mg}$ protein) within about the same range as in the hypothalamus. Left atrial concentration was $1,739 \pm 16 \mathrm{pg} / \mathrm{mg}$ protein. Ventricular OT levels were lower in comparison to those of the atria. In addition, in vitro studies showed OT secretion from atrial myocytes 
and the presence of OT in heart perfusates. Specific OT transcripts were demonstrated in all four heart compartments by PCR amplification of rat heart cDNA. The amplified fragment of the OT gene generated from rat heart chambers was identical in size to that of the uterus. This finding indicates that OT transcripts are structurally identical in the heart and uterus.

Since we have discovered that OT is produced and released by the heart and acts on its cardiac receptors to decrease heart rate and force of contraction, we hypothesized that OT might be generated in the vasculature and dilate vessels. Indeed, our studies showed that an intrinsic OT system sensitive to estrogenic regulation exists in the vasculature (58). Immunoreactive OT has been identified in rat, sheep and dog vessels by specific radioimmunoassay, and characterized by HPLC. RT-PCR analysis identified OT transcripts containing the coding sequence of the OT gene in the aorta and vena cava. Membrane binding with radiolabeled OT antagonist and detection of OTR mRNA confirmed the presence of OTR in the vasculature. A dose of estrogen that mimics the concentrations in rats in early pregnancy enhanced OT and OTR gene expression in the vena cava, but OTR mRNA was increased only in the aorta (59).

We have now discovered not only the presence of functional OTR in the various chambers of the heart with a higher number in the right atria than in other chambers, but we also have found that $\mathrm{OT}$ is resident within the heart and can be synthesized there. Consequently, not only would OT reach the heart by the circulation to induce ANP release, but also intracardiac OT might play a role in stimulating ANP release (Figure 2).

Furthermore, OTR have been found in the kidney, and our recent studies indicate that the OT released by volume expansion acts not only to release ANP that produces natriuresis by action on its receptors in the kidney, but also acts on its receptors on nitric oxide synthase (NOS)-containing renal tubular cells to activate nitric oxide (NO) release (60). Both ANP and NO activate guanylyl cyclase to convert guanosine 5'triphosphate to monophosphate (cGMP), that augments natriuresis by closing sodium channels. Although it has not yet been proven, it is likely that OT also acts on its receptors in the heart to activate NOS to release NO that may act in conjunction with ANP to decrease the rate and force of contraction of the heart, also by increasing cGMP.

Since the OT antagonist blocked the negative chrono- and inotropic effects of OT, we believe that local OT production in the heart may be physiologically relevant by releasing ANP through the activation of OTR. The released ANP slows the heart and reduces its force of contraction by intracardiac action. Implications of the OT system in the large vessels may include local $\mathrm{C}$ natriuretic peptide and ANP formation, which, consequently, induces vasorelaxation (59). The indirect effect of OT by induction of the release of $\mathrm{C}$ natriuretic peptide or ANP may

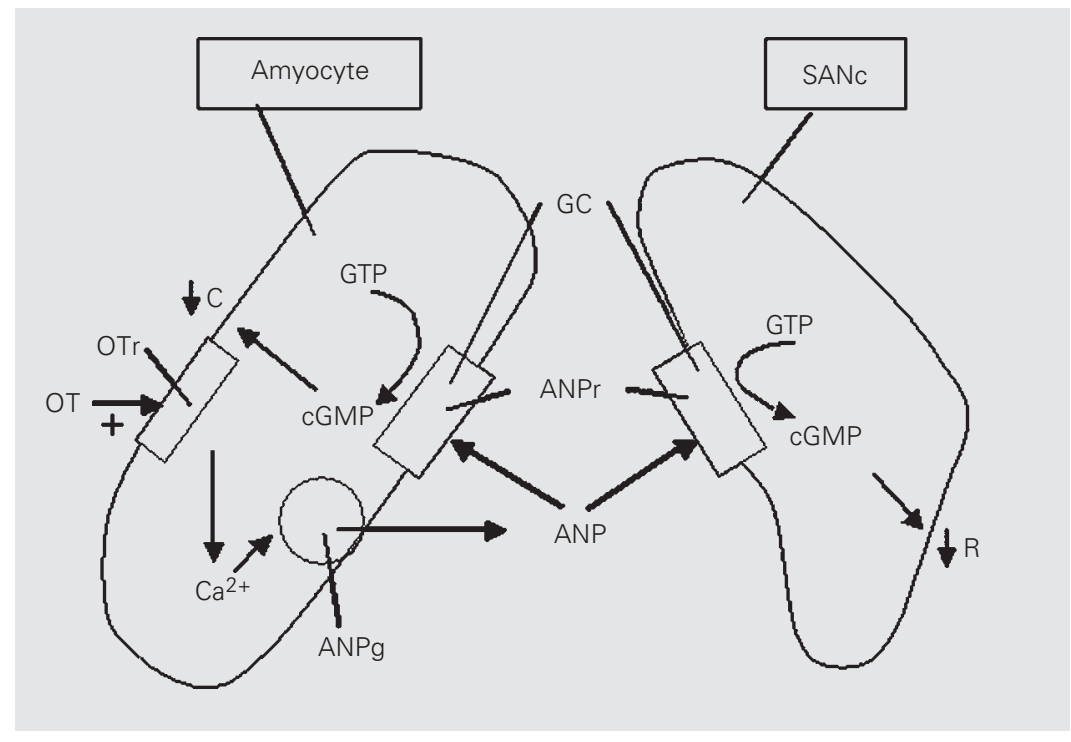

Figure 2. Schematic diagram of the proposed mechanism of oxytocin-induced atrial natriuretic peptide (ANP) release in the right atrium. For a detailed description, see Discussion. Amyocyte, atrial myoocyte; SANc, sinoatrial node cell; OT, oxytocin; OTr, oxytocin receptor; ANPg, ANP secretory granule; ANPr, ANP receptor; GC, guanylyl cyclase; GTP, guanosine5'-triphosphate; cGMP, cyclic guanosine-3',5'-monophosphate; C, contraction; R, heart rate; short arrow down, decrease; plus (+), activation (from Ref. 55, with permission). 
inhibit growth and cell proliferation (59).

In conclusion, these results support the concept that OT and ANP act in concert to control body fluid and cardiovascular homeostasis. The actions of both peptides are mediated by the activation of guanylyl cyclase and generation of cGMP. In the kidney, and probably in the heart and vasculature as well, OT works in part by NOS activation that releases NO, which also activates guanylyl cyclase and generates cGMP. Therefore, cGMP is the common mediator of vascular relaxation and possibly of negative inotropic and chronotropic effects in the heart, as well as natriuresis.

\section{Hypothalamic control of salt intake}

As described in the Introduction, it has been known since the early 1950's that stimulation of the hypothalamus by microinjection of hypertonic saline or electrical stimulation evokes drinking, whereas lesions in the same areas produce adipsia. Subsequent studies showed a stimulatory role of acetylcholine in drinking and facilitatory effects of catecholamines as well. AII is the most potent dipsogenic substance known, whereas OT and ANP have inhibitory effects on drinking. Indeed, the actions of AII are opposed by ANP and OT also has opposing actions on water and salt intake and excretion. Within the brain ANP inhibits drinking induced by AII and blocks dehydration-induced drinking that is known to be caused by the release of AII. $\alpha$-Adrenergic agonists are known to release ANP and antagonize AII-induced drinking. We examined the hypothesis that $\alpha$-agonists block AII-induced drinking by stimulating the release of ANP from ANPsecreting neurons (ANPergic neurons) within the brain that inhibit the effector neurons stimulated by AII to induce drinking (61). Injection of AII (12.5 ng) into the AV3V, at the effective dose to increase water intake, increased plasma ANP concentrations $(\mathrm{P}<0.01)$ within $5 \mathrm{~min}$. Previous injection of phenylephrine (an $\alpha_{1}$-adrenergic agonist) or clonidine (an $\alpha_{2}$-adrenergic agonist) into the AV3V region significantly reduced AII-induced water intake. Their injection also induced a significant increase in plasma ANP concentration and in ANP content in the olfactory bulb, AV3V, medial basal hypothalamus and ME. These results suggest that the inhibitory effect of both $\alpha$-adrenergic agonists on AII-induced water intake can be explained, at least in part, by the increase in ANP content and presumed release from these neural structures. The increased release of ANP from the axons of neurons terminating on the effector neurons of the drinking response by stimulation of ANP receptors would inhibit the stimulatory response evoked by the action of AII on its receptors on these same effector neurons.

Thus, within the brain ANP and OT act together to inhibit drinking and sodium intake. They also act in concert on the heart and vasculature to produce a rapid decrease in circulating blood volume. Finally, blood volume is returned to normal by the combined natriuretic action of circulating OT acting on the kidney via activation of $\mathrm{NO}$ release with consequent cGMP release, and by circulating ANP acting on its receptors to also release cGMP. The cGMP closes sodium channels thereby having a natriuretic effect and finally returning blood volume to normal. At least with regard to water and salt intake, but possibly also with regard to the other actions of OT and ANP, these actions may be opposed by AII.

\section{Conclusion}

In conclusion, it is now clear that AII and ANP in the brain play important and opposite roles in the control of water and salt intake, with AII promoting the intake of both and ANP inhibiting the intake of both, perhaps by inhibiting AII secretion within the brain. In fact, there is probably a reciprocal relationship between the activity of these 
two neuronal systems. Again, on the output side, the two are related since ANP release is stimulated in situations of volume expansion to increase renal sodium and potassium loss, and at the same time ANP not only blocks the release of renin from the kidney, thereby decreasing AII stimulation of aldosterone release, but also directly inhibits aldosterone secretion. Decreased aldosterone secretion results in increased natriuresis. ANP neurons in the hypothalamus inhibit the release of corticotropin-releasing factor and VP, thereby decreasing adrenocorticotrophic hormone, and thus reducing aldosterone secretion.

Therefore, both of these peptides play a key role in the maintenance of body fluid homeostasis in the body. ANP acts slowly via natriuresis to reduce effective circulating blood volume. The rapid release of ANP following BVE would first produce decreased heart rate, force of contraction and vasodilation by activating particulate guanylate cyclase, leading to the production of cGMP, which by activating protein kinase $G$ would induce these cardiovascular effects. OT also plays a crucial role in the response to BVE. Baroreceptor-induced release of ANP induces OT release. The OT circulates to the heart and acts on its receptors there to induce ANP release producing a reduction in heart rate and force of contraction that, coupled with ANP-induced vasodilation, causes a rapid reduction in effective circulating blood volume. The intrinsic OT system in the heart and vascular system augments the effects of circulating OT. These combined actions would result in a rapid reduction in the effective circulating blood volume. The longer term effects would be mediated not only by diuresis and natriuresis but also by decreased intake of salt and water.

\section{References}

1. Andersson B \& McCann SM (1954). A further study of polydipsia evoked by hypothalamic stimulation in the goat. Acta Physiologica Scandinavica, 33: 333-346.

2. Andersson B \& McCann SM (1955). Drinking, antidiuresis and milk ejection from electrical stimulation within the hypothalamus of the goat. Acta Physiologica Scandinavica, 35: 313-320.

3. Zimmerman EA (1983). Oxytocin, vasopressin and neurophysins. In: Kreiger DT, Brownstein MJ \& Martins JB (Editors), Brain Peptides. John Wiley \& Sons, New York, NY, USA, 598-611.

4. Palkovits M, Eskay RL \& Antoni FA (1987). Atrial natriuretic peptide in the median eminence is of paraventricular nucleus origin. Neuroendocrinology, 46: 542-544.

5. Andersson B \& McCann SM (1956). The effects of hypothalamic lesions on the water intake of the dog. Acta Physiologica Scandinavica, 36: 312-320.

6. Witt DM, Keller AD, Batsel HL \& Lynch JR (1952). Absence of thirst and resultant syndrome associated with anterior hypothalamectomy in the dog. American Journal of Physiology, 171: 780 (Abstract).

7. Welt LG, Seldin DW, Nelson WP, German WJ \& Peters JP (1952). Role of central nervous system in metabolism of electrolytes and water. Archives of Internal Medicine, 90: 355-356.

8. McCann SM, Franci CR \& Antunes-Rodrigues J (1989). Hormonal control of water and electrolyte intake and output. Acta Physiologica Scandinavica, 136 (Suppl 583): 97-104.

9. Andersson B, Jobin M \& Olsson K (1966). Stimulation of urinary salt excretion following injections of hypertonic $\mathrm{NaCl}$ solution into the third brain ventricle. Acta Physiologica Scandinavica, 67: 127-128.

10. Dorn J \& Porter JC (1970). Diencephalic involvement in sodium excretion in the rat. Endocrinology, 78: 1112-1117.
11. Antunes-Rodrigues J \& McCann SM (1970). Chemical stimulation of water, sodium chloride and food intake by injections of cholinergic and adrenergic drugs into the third brain ventricle. Proceedings of the Society for Experimental Biology and Medicine, 133: 1464-1470.

12. Grossman SP (1969). A neuropharmacological analysis of hypothalamic and extrahypothalamic mechanisms concerned with the regulation of food and water intake. Annals of the New York Academy of Sciences, 157: 902-917.

13. Dorn J, Antunes-Rodrigues J \& McCann SM (1970). Natriuresis in the rat following intraventricular carbachol. American Journal of Physiology, 219: 1292-1298.

14. Andersson B (1977). Regulation of body fluids. Annual Review of Physiology, 39: 185-200.

15. Morris M, McCann SM \& Orias R (1977). Role of transmitters in mediating hypothalamic control of electrolyte secretion. Canadian Journal of Physiology and Pharmacology, 55: 1143-1154.

16. Petrovic SL, McDonald JK, Bedran de Castro JC, Snyder GD \& McCann SM (1985). Regulation of anterior pituitary and brain betaadrenergic receptors by ovarian steroids. Life Sciences, 37: 15631570.

17. Covian MR, Antunes-Rodrigues J, Gentil CG, Saad WA, Camargo LAA \& Silva-Netto CR (1975). Central control of salt balance in neural integration of physiological mechanisms and behavior. In: Mogenson CJ \& Calaresu FR (Editors), Neural Integration of Physiological Mechanisms and Behavior. University of Toronto Press, Toronto, Canada, 267-282.

18. Covian MR \& Antunes-Rodrigues J (1963). Specific alterations in sodium chloride intake after hypothalamic lesions in the rat. American Journal of Physiology, 205: 922-926. 
19. DeWardener HE \& Clarkson EL (1985). Natriuretic hormone. In: Seldin DW \& Giebisch G (Editors), The Kidney: Physiology and Pathophysiology. Raven Press, New York, NY, USA, 1013-1031.

20. Davis JO \& Freeman RH (1976). Mechanisms regulating renin release. Physiological Reviews, 56: 1-56.

21. Cort JH, Lichardus B, Pliska V, Barth T, Uhrin V \& Rudinger J (1969). A hypothalamic natriuretic peptide. In: Margoulies JC (Editor), Protein and Polypeptide Hormones. Excerpta Medica, Amsterdam, The Netherlands.

22. Orias R \& McCann SM (1970). Natriuretic effect of $\alpha-M S H$ in the water-loaded rat. Proceedings of the Society for Experimental Biology and Medicine, 133: 469-474.

23. Orias R \& McCann SM (1972). Natriuresis induced by alpha and beta melanocyte stimulating hormone (MSH) in rats. Endocrinology, 90: 700-706.

24. Morris M, McCann SM \& Orias R (1976). Evidence for hormonal participation in the natriuretic and kaliuretic responses to intraventricular hypertonic saline and norepinephrine. Proceedings of the Society for Experimental Biology and Medicine, 152: 95-98.

25. Orias R \& McCann SM (1972). Intraventricular injection of hypertonic saline induces natriuresis in rats with hereditary diabetes insipidus. Proceedings of the Pan American Physiological Congress, Caracas, Venezuela.

26. DeBold AJ, Bovenstein HB, Veress AT \& Sonnenberg H (1981). A rapid and potent natriuretic response to intravenous injection of atrial myocardial extract in rats. Life Sciences, 28: 89-94.

27. Gauer OH \& Henry JP (1963). Circulatory phases of fluid volume control. Physiological Reviews, 43: 423-481.

28. Synhorst DP \& Gutkowska J (1988). Atrial distension of isolated rabbit hearts and release of ANP. American Journal of Physiology, 255: R232-R236.

29. Samson WK (1985). Dehydration-induced alterations in rat brain vasopressin and atrial natriuretic factor immunoreactivity. Endocrinology, 117: 1279-1281.

30. Blaine EH \& Rosenblatt M (1987). ANF: A hormone from the heart. In: McCann SM (Editor), Endocrinology: People and Ideas. Oxford University Press, Cary, NC, USA, 397-420.

31. Skofitsch G, Jacobowitz DM, Eskay RL \& Zamir N (1985). Distribution of ANF-like immunoreactive neurons in the rat brain. Neuroscience, 16: 917-948.

32. Antunes-Rodrigues J, McCann SM, Rogers LC \& Samson WK (1985). Atrial natriuretic factor inhibits water intake in conscious rats. Proceedings of the National Academy of Sciences, USA, 82: 8720-8724.

33. Antunes-Rodrigues J, McCann SM \& Samson WK (1986). Central administration of atrial natriuretic factor inhibits salt intake in the rat. Endocrinology, 118: 1726-1729.

34. Franci CR, Kozlowski GP \& McCann SM (1989). Water intake in rats submitted to hypothalamic immunoneutralization of angiotensin II, atrial natriuretic peptide, vasopressin or oxytocin. Proceedings of the National Academy of Sciences, USA, 86: 2952-2956.

35. Malvin RLM, Mouwd D \& Vander AJ (1977). Angiotensin: Physiological role in water deprivation-induced thirst of rat. Science, 197: 171-173.

36. Phillips MI (1987). Functions of angiotensin in the central nervous system. Annual Review of Physiology, 49: 413-435.

37. Brody MJ \& Johnson AK (1980). Role of the anteroventral third ventricle region in fluid and electrolyte balance, arterial pressure regulation, and hypertension. In: Martini L \& Ganong WF (Editors), Frontiers of Neuroendocrinology. Raven Press, New York, NY, USA, 249-292.

38. Baldissera S, Menani JV, Sotero dos Santos LF, Favaretto ALV,
Gutkowska J, Turrin MQA, McCann SM \& Antunes-Rodrigues J (1989). Role of the hypothalamus in the control of atrial natriuretic peptide release. Proceedings of the National Academy of Sciences, USA, 86: 9621-9625.

39. Antunes-Rodrigues J, Ramalho MJ, Reis LC, Menani JV, Turrin MQA, Gutkowska J \& McCann SM (1991). Lesions of the hypothalamus and pituitary inhibit volume-expansion-induced release of atrial natriuretic peptide. Proceedings of the National Academy of Sciences, USA, 88: 2956-2960

40. Gutkowska J, Antunes-Rodrigues J \& McCann SM (1997). Atrial natriuretic peptide in brain and pituitary gland. Physiological Reviews, 77: 465-515

41. Antunes-Rodrigues J, Picanço-Diniz DWL, Favaretto ALV, Gutkowska J \& McCann SM (1993). Brain atrial natriuretic peptide neurons play an essential role in volume expansion-induced release of atrial natriuretic peptide and natriuresis. Neuroendocrinology, 58: 696-700.

42. Charles J, Tang F, Cameron VA, Richards AM \& Espiner EA (1991) Intracerebral ANF antiserum inhibits volume-induced ANF in sheep. Endocrinology, 129: 2225-2230

43. Antunes-Rodrigues J, Marubayashi U, Favaretto ALV, Gutkowska J \& McCann SM (1993). Essential role of hypothalamic muscarinic and $\alpha$-adrenergic receptors in atrial natriuretic peptide release induced by blood volume expansion. Proceedings of the National Academy of Sciences, USA, 90: 10240-10244.

44. Antunes-Rodrigues J, Machado BH, Andrade HA, Mauad H, Ramalho MJ, Reis LC, Silva-Netto CR, Favaretto ALV, Gutkowska J \& McCann SM (1992). Carotid-aortic and renal baroreceptors mediate the atrial natriuretic peptide release induced by blood volume expansion. Proceedings of the National Academy of Sciences, USA, 89: 68296831.

45. Morris M \& Alexander N (1988). Baroreceptor influences on plasma atrial natriuretic peptide (ANP): sinoaortic denervation reduces basal levels and the response to an osmotic challenge. Endocrinology, 122: $373-375$

46. Bosler O \& Descarries L (1987). Monoamine innervation of the organum vasculosum laminae terminalis (OVLT): A high resolution radioautographic study in the rat. Journal of Comparative Neurology, 272: $545-561$

47. Stein JM, Lind RW \& Johnson AK (1987). Central serotonergic influences on renal electrolyte and water excretion. Neuropharmacology, 26: 1685-1692.

48. Reis LC, Ramalho MJ \& Antunes-Rodrigues J (1991). Participation of the median raphe nucleus and central serotoninergic pathways in the control of water electrolyte excretion. Brazilian Journal of Medical and Biological Research, 24: 847-854

49. Reis LC, Ramalho MJ, Favaretto ALV, Gutkowska J, McCann SM \& Antunes-Rodrigues J (1994). Participation of the ascending serotonergic system in the stimulation of atrial natriuretic peptide release. Proceedings of the National Academy of Sciences, USA, 91: 1202212026.

50. Antunes-Rodrigues J, Ramalho MJ, Reis LC, Picanço-Diniz DWL, Favaretto ALV, Gutkowska J \& McCann SM (1993). Possible role of endothelin acting within the hypothalamus to induce the release of atrial natriuretic peptide and natriuresis. Neuroendocrinology, 58: 701-708.

51. Haanwinckel MA, Elias LK, Favaretto ALV, Gutkowska J, McCann SM \& Antunes-Rodrigues J (1995). Oxytocin mediates atrial natriuretic peptide release and natriuresis after volume expansion in the rat. Proceedings of the National Academy of Sciences, USA, 92: 7902-7906 
52. Martin DR, Perahouse JB, Trigg DJ, Vesely DL \& Buerkert JE (1990). Three peptides from ANF prohormone $\mathrm{NH}$ 2-terminus are natriuretic and/or kaliuretic. American Journal of Physiology, 258: F1401-F1408.

53. Vesely DF, Norsk P, Gower WR, Chiou S \& Epstein M (1995). Release of kaliuretic peptide during immersion-induced central hypervolemia in healthy humans. Proceedings of the Society for Experimental Biology and Medicine, 209: 20-26.

54. Favaretto ALV, Ballejo GO, Albuquerque-Araujo WIC, Gutkowska J, Antunes-Rodrigues J \& McCann SM (1997). Oxytocin releases atrial natriuretic peptide from rat atria in vitro that exerts negative inotropic and chronotropic action. Peptides, 18: 1377-1381.

55. Gutkowska J, Jankowski M, Lambert C, Mukaddam-Daher S, Zingg $\mathrm{HH}$ \& McCann SM (1997). Oxytocin releases atrial natriuretic peptide by combining with oxytocin receptors in the heart. Proceedings of the National Academy of Sciences, USA, 94: 11704-11709.

56. Gutkowska J, Jankowski M, Mukaddam-Daher S \& McCann SM (2000). Oxytocin is a cardiovascular hormone. Brazilian Journal of Medical and Biological Research, 33: 625-633.

57. Jankowski M, Hajjar F, Al Kawas S, Mukaddam-Daher S, Hoffman G, McCann SM \& Gutkowska J (1998). Rat heart: A site of oxytocin production and action. Proceedings of the National Academy of Sciences, USA, 95: 14558-14563.

58. Jankowski M, Rachelska G, Donghao W, McCann SM \& Gutkowska $J$ (2001). Estrogen receptors activate atrial natriuretic peptide in the rat heart. Proceedings of the National Academy of Sciences, USA, 98: 11765-11770.

59. Jankowski M, Wang D, Hajjar F, Mukaddam-Daher S, McCann SM \& Gutkowska J (2000). Oxytocin and its receptors are synthesized in the rat vasculature. Proceedings of the National Academy of Sciences, USA, 97: 6207-6211.

60. Soares TJ, Coimbra TM, Martins AR, Pereira AGF, Carnio EC, Branco LGS, Albuquerque-Araujo WIC, de Nucci G, Favaretto ALV, Gutkowska J, McCann SM \& Antunes-Rodrigues J (1999). Atrial natriuretic peptide and oxytocin induce natriuresis by release of cGMP. Proceedings of the National Academy of Sciences, USA, 96: 278-283.

61. Bastos R, Favaretto ALV, Gutkowska J, McCann SM \& AntunesRodrigues J (2000). Alpha-adrenergic agonists inhibit the dipsogenic effect of angiotensin II by their stimulation of atrial natriuretic peptide release. Brain Research, 895: 80-88. 